\title{
Evaluación del desempeño profesoral en Educación Superior Tecnológica
}

\author{
Mary Susana Mantilla Falcón \\ Ingeniera en Gestión Comercial \\ Docente, Instituto Superior Tecnológico Edupraxis, Ecuador \\ mmantilla@tecnologicoedupraxis.com
}

Recepción: 10-04-2019 / Aceptación: 03-08-2019

\section{Resumen}

El desempeño laboral de los profesores constituye un medio poderoso para mejorar la calidad educativa; en tal sentido, la evaluación del mismo es un proceso que sirve para juzgar la excelencia y las cualidades de una persona y, sobre todo, su contribución al logro de los objetivos de la organización.

En esta investigación se analizó la evaluación del desempeño de 49 profesores de la carrera de Administración de Empresa del Instituto Superior Tecnológico Edupraxis en Ecuador. Para el caso de estudio, los criterios predominantes fueron los didácticos. De ahí que se sugiere una propuesta de criterios de evaluación profesoral, que consideren como elemento fundamental un plan de mejoramiento profesional.

Palabras clave: Desempeño, evaluación, profesores, educación tecnológica.

\begin{abstract}
Professors' job performance constitutes a powerful means of improving the educational quality; In that sense, its evaluation is a process that serves to judge the excellence and qualities of a person and, above all, his or her contribution to achieving the objectives of the organization.

In this research, the performance evaluation of 49 professors of the major of Business Administration of the Higher Technological Institute Edupraxis in Ecuador was analyzed. For the case study, the predominant criteria were didactic. Hence that a proposal for professor evaluation criteria is suggested, which considers a professional improvement plan as a fundamental element.
\end{abstract}

Keywords: Performance, evaluation, professors, technological education. 


\section{Introducción}

Una de las funciones fundamentales de la gestión del talento humano es la evaluación del desempeño docente, por cuanto puede asegurar la búsqueda de la excelencia para que la organización sea competitiva, y obtenga los resultados deseados en el mundo globalizado de hoy. En este marco, las instituciones de educación superior vienen generando una cultura de evaluación centrada en la mejora, donde los docentes son piezas clave en la formación de alto nivel de los futuros profesionales requeridos, para alcanzar la tan ansiada sociedad del conocimiento. En tal sentido, se hacen esfuerzos por garantizar la evaluación profesoral.

Los cambios generados por la revolución informática y comunicacional han dado lugar a los que variadísimos autores, entre los que destacan Touraine (1973), Bell (1991), Drucker (1993), Barceló (1998), Tedesco (1999) y Hargreaves (2007), entre otros, han denominado la sociedad del conocimiento; que es la expresión de la conmoción generada en la percepción, la imaginación y el pensamiento contemporáneo, por la actual revolución científico - digital y la globalización. La premisa fundamental de esta nueva sociedad es que el conocimiento y la información son actualmente el único factor de producción, dejando a un lado las tradicionales: trabajo, tierra y capital. Es indudable que la naturaleza del trabajo está cambiando. En consecuencia, hasta el trabajo de más baja catego- ría pasa a ser un trabajo de conocimiento; significa conocer el entorno competitivo y el hacer de la organización.

Lo planteado ha impactado la gestión de personas en el sentido que es cada vez más clara su importancia estratégica. Gallardo y Zampela (2001) señalan que se asume como un área multidisciplinaria, formando profesionales con una metodología científica, pero que a la vez puedan resolver problemas prácticos surgidos en la organización. Una de sus funciones fundamentales es la evaluación del desempeño, por tanto, Chiavenato (2004) señala que "el desempeño humano debe ser excelente en todo momento para que la organización sea competitiva y obtenga resultados en el mundo globalizado de hoy" (p.147). Su evaluación constituye un medio poderoso para resolver problemas de desempeño, mejorar la calidad del trabajo y la calidad de vida en las organizaciones.

No obstante, para Martínez-Izaguirre y otros (2018), una problemática particular en el proceso de evaluación del desempeño en los docentes de educación superior es que no se les considera como "sujeto activo en el análisis de su realidad y menos en la construcción de senderos hacia su mejora" (p.4). A tales planteamientos no escapan los institutos de tecnología, en donde la gestión de personas se sigue manejando como una función convencional. Al respecto, Álvarez (2003) señala que la selección, contratación, evaluación y desarrollo de personal se realiza bajo criterios 
tradicionales. En cuanto a la evaluación es -por lo general- unidireccional, en función de la opinión de los superiores.

López (2014) señala que la evaluación del desempeño del docente en Ecuador -aunque no generalizada, ni obligatoria- ha venido aplicándose desde hace varios años en las universidades, preferentemente con cuestionarios aplicados a los estudiantes. Con el propósito de mejorar esta situación, la Ley Orgánica de Educación Superior (2010) y el Reglamento de Carrera y Escalafón del Docente e Investigador del Sistema de Educación Superior (2012) se ocupan ampliamente de la evaluación docente, y contemplan los criterios y mecanismos para dicho proceso.

Así, en el Instituto de Tecnología Edupraxis en Ecuador, si bien existen instrumentos de evaluación del desempeño docente actualizados, estos son administrados de acuerdo a la opinión individualizada de los diferentes directivos; ya que no hay una unidad particular que realice esta función. Es un proceso normativo que se realiza solo por cumplir $y$, por ende, con cierta improvisación; tampoco se consideran planes de mejora. En tal sentido, para Quintero (2019) "una evaluación del desempeño sin una retroalimentación pierde sentido y esencia, lamentable es común ver en las instituciones públicas y privadas aplicar formatos [...] y no considerar a la evaluación una herramienta útil para el perfeccionamiento [...] (p.17).

Si se intenta buscar la causa de tal problemática es posible encontrarlas en la resistencia al cambio de directivos y profesores; lo cual es explicable en el hecho que el cambio, aún el positivo produce sensación de miedo. De acuerdo a Chiavenato (2004), el cambio en las organizaciones no solo trastorna los esquemas, sino que, con frecuencia, implica que se deben hacer cosas para las cuales no se está preparando. En el caso particular, es también posible que tanto directivos como profesores no tengan un conocimiento profundo de las nuevas tendencias de la evaluación del desempeño; y aun conociéndolas, no tengan los métodos y herramientas adecuados para implementar los cambios.

Esta situación se agrava en la institución en la cual se realizó el caso de estudio, por cuanto existiendo cierta continuidad en la contratación, los profesores sienten estabilidad laboral y posiblemente descuidan la búsqueda de mejoras e innovaciones en su desempeño. $\mathrm{O}$, por el contrario, la institución desconozca los verdaderos aportes de muchos de estos profesionales. Por esta razón se hace necesario evaluar el desempeño de estos profesores de tal manera que contribuya a estar a la vanguardia, ayudando a la institución a hacer los cambios necesarios.

En este aspecto, la evaluación de profesores constituye un reto frente a la necesidad de optimizar las instituciones de Educación Superior de Tecnología, en la búsqueda constante y permanente del incremento de la calidad. De ahí que el objetivo del estudio fue analizar el desempeño de los docentes en el Instituto de Tecnología Edupraxis en Ecuador. 
Al respecto, Mora (2004) señala que, en el mundo académico, el término de evaluación ha estado relacionado con la idea de medir y establecer juicios de valor con respecto al desempeño de una persona. Esta concepción de evaluación se puede calificar de simple, reduccionista, mecánica, previsible. Frente al fracaso y las limitaciones de este modelo positivista de la evaluación surge la actual tendencia a considerar otros enfoques en la evaluación del desempeño. De esta forma se pretende conocer cómo están realizando su labor los profesores; y de este modo destacar sus fortalezas y capacidades, además de reconocer sus debilidades y las amenazas a las que se deben enfrentar en la gestión académica. De acuerdo con Hidalgo (2007), la evaluación del desempeño académico es "un proceso social, constructivo e investigativo vinculado al acto pedagógico que incluye la participación de todos sus autores [...] (p.14).

Como se puede ver, la evaluación del desempeño es una valoración de la actuación del profesor de manera sistemática, a fin de mejorar la calidad de la práctica educativa. Evaluar se convertirá entonces, según Marcano (2008), en un proceso de discusión y reflexión en el que el cambio y la transformación del sujeto sean efectivos para lograr el desarrollo del potencial creativo y de la personalidad del individuo. El desempeño, por su parte, puede definirse como las tareas específicas que el profesor debe cumplir a lo largo del curso, y en un tiempo estipulado, relacionarlas con cómo dirigir correctamente el aprendizaje en su forma más general.
Existe una gran variedad de criterios y juicios para considerar que es un desempeño adecuado. Al respecto, Chavarry (2009) plantea que en la evaluación de este se consideran las competencias docentes, investigativas, didácticas, académicas, ética, comunicativa, cultural y tecnológica; lo cual es una propuesta integradora al considerar las competencias profesionales del profesor de educación superior como expresión de las diferentes aristas y esferas de su actuación.

Por su parte, Lawler III (2000) plantea que el proceso de evaluación del desempeño debe ser un proceso continuo que se prolongue en el tiempo. Asimismo, sus actividades deben diseñarse de manera que las personas perciban que son evaluadas periódicamente, con justicia y de manera integral. En este sentido, Davis (1992) plantea que existen tres procedimientos para realizar la evaluación del desempeño profesoral:

1. Los logros alcanzados por el estudiante; aparentemente debería existir una relación directa entre la eficiencia del profesor y el éxito alcanzado por los estudiantes.

2. Instrumentos estandarizados que midan la competencia docente.

3. Opinión de los estudiantes, que es uno de los procedimientos más importantes en esta actividad y el que ofrece mayor polémica, ya que se afirma que estos no están lo suficientemente capacitados para juzgar la competencia de un profesor. 
Para Coppola (2012) hay tres grandes tendencias de la evaluación de la función docente universitaria:

a. Orientada a resultados relacionados al control o rendición de cuentas.

b. Encaminada a la formación, donde aparece como un proceso educativo sistemático y permanente integrada a la actividad cotidiana de la docencia, operando en el interior de las cátedras y contribuyendo a mejorarlas.

c. La relacionada con políticas institucionales para el desarrollo del talento humano, vinculada con la carrera docente y su relación con el ingreso, formación permanente, promoción, salario, incentivos e investigación.

En este aspecto, algunos antecedentes de la temática abordada se presentan seguidamente en orden cronológico desde los más cercanos a los más lejanos:

- Lima (2017) realizó un trabajo con el fin de determinar la relación entre evaluación del desempeño en el aula de los docentes y el rendimiento académico de los estudiantes de la carrera de Ingeniería Metalúrgica y Materiales en la Universidad Mayor de San Andrés, La Paz - Ecuador. Para esto se realizó una investigación de tipo descriptivo y correlacional, con un diseño no experimental transeccional, en un enfoque paradigmático positivista.
La muestra fue no probabilística: se abordó a 15 docentes y 125 estudiantes que cursaron las asignaturas durante el período I-2007. Se aplicó una encuesta con base a una escala de medición de actitudes tipo Likert de 34 ítems para la evaluación del desempeño docente en el aula, cuyos resultados evidenciaron un desempeño docente bueno. Por su parte, el rendimiento académico se midió a través de las calificaciones finales, lo que permitió clasificarlo como regular. Para el análisis de los datos se utilizó el programa SPSS, mostrando una relación significativa de fuerza baja entre las dos variables estudiadas. De ahí que se propone complementar con otras formas la evaluación del profesor y crear conciencia en los estudiantes para que den información fidedigna, sin presión docente o por otros factores subjetivos.

- Por su parte, Barreto (2015) realizó una investigación acerca del desempeño docente y el desarrollo de las capacidades en gestión empresarial y marketing en estudiantes de Lima. Fue un diseño no experimental, explicativo causal, de nivel correlacional. A efecto de recoger la información, se aplicaron dos encuestas a 80 estudiantes y 20 docentes del IV ciclo, en la asignatura Gestión empresarial y marketing de la carrera Edificaciones de la Escuela Superior Tecnológica -SINCICO en el año 2014. El tratamiento de la información se hizo mediante la distribución de frecuencia media y la prueba de Chi Cuadrado. 
Se logró demostrar que el desempeño docente influyo en la capacidad de gestión de aprendizaje de los estudiantes, por lo que se aceptó la hipótesis. De ahí que se recomienda capacitar a los docentes para mejorar su desempeño en la metodología de la enseñanza; y en el uso de materiales didácticos acordes con el avance de la ciencia y la tecnología, que se verá reflejado en el desempeño de los estudiantes.

- En el mismo orden de ideas, Arias (2015) realizó una investigación cuyo propósito fue diseñar y utilizar un manual de evaluación para determinar los niveles de desempeño de los formadores de docentes en las diferentes especialidades que trabajan en doce Instituciones de Educación Superior en El Salvador. Para recoger la información se aplicaron tres cuestionarios tipo escala, con cuatro alternativas: el primero, a 50 coordinadores; el segundo, a 869 estudiantes, y el tercero -de autoevaluación- a 262 docentes de la muestra.

El tratamiento de la información se hizo con ayuda del programa SPSS, permitiendo concluir que, según el 55.9\% de los coordinadores, el $55.6 \%$ de los docentes y el $46 \%$ de los estudiantes realiza evaluación de procesos generales, pero no se valora el desempeño específico; por lo que no se ha logrado una valoración a nivel del país acerca del desempeño de los formadores de docentes. De ahí que se considera muy importante que el Ministerio de Educación (MINED) implemente un proceso de evaluación, cuyo objetivo más importante esté orientado a diagnosticar deficiencias e implementar planes de mejoramiento de la calidad educativa de forma periódica. Por otra parte, debe crearse una cultura de evaluación.

- Por su parte, López (2014) hizo un diagnóstico del sistema de evaluación del desempeño, para proponer uno alternativo, fundamentándose en la evaluación participativa. Fue un estudio tipo proyecto factible, con base a una investigación de campo, con una muestra de 3 directivos, 20 docentes y 74 estudiantes a los cuales se les aplicó una encuesta. Se comprobó que el actual sistema de evaluación, según el $60 \%$ de los encuestados, presenta debilidades entre las cuales se puede mencionar la ausencia de comisiones encargadas de la evaluación del desempeño docente. Se plantea considerar la opinión del estudiante (heteroevaluación), la coevaluación con la opinión de pares y la autoevaluación.

- Coppola (2012) realizó un estudio ubicado en el paradigma cualitativo, comparando las políticas de evaluación de la función docente universitaria en tres países: Argentina, España y México a partir de diversas fuentes de conocimiento y de las reflexiones de los docentes. Se aplicaron 40 entrevistas con base a un guión de nueve preguntas a 20 administrativos, 10 estudiantes y 10 profesores. También se realizaron tres grupos focales, uno en cada país participante, con base en un guion de diez preguntas.

En la fase documental se encontró que, en Argentina, el tema aún no tiene desarrollo; 
en México, si bien existe mayor producción, incluso existe una red de evaluación de la docencia desde hace más de diez años, hay una notable sistematización de datos. En España se ha podido acceder con cierta facilidad a la documentación estadística y de la agencia de evaluación.

Se realizó la categorización de la información obtenida de las entrevistas y grupos focales, siguiendo las pautas del método comparativo constante, utilizando el software Atlas Ti. Se concluyó que la evaluación de la función docente universitario -como parte de una evaluación institucional- es un proceso de múltiples dimensiones y contradicciones; por lo que debería ser tratada por las teorías de la complejidad, y ejercitada mediante el uso de diversos instrumentos y la combinación de diferentes abordajes.

- Por su parte, Barreto (2012) realizó una investigación para evaluar la calidad del desempeño docente y directivo. Se hizo en el Colegio Técnico Superior Agropecuario José Benigno Iglesias, en Loja-Ecuador, en la sección nocturna. La muestra fue de 88 personas (nueve docentes, un directivo, 73 estudiantes, tres miembros del Consejo Técnico y el supervisor escolar).

La investigación se ubicó en el paradigma de análisis crítico, el método inductivo- deductivo y analítico. Para recoger la información se aplicaron cuatro cuestionarios: autoevaluación de los profesores, evaluación de los estudiantes, evaluación por parte de los directivos y la observación de una clase de cada docente.
El tratamiento de la información se hizo mediante la estadística descriptiva.

Se pudo concluir que el actual desempeño docente y directivo es bueno; siendo la mayor debilidad la poca vinculación con la comunidad, escaso uso de la tecnología y falta de investigación. De ahí que se recomienda asumir la evaluación como política institucional y no como obligación ministerial. En tal sentido, la evaluación de docentes y directivos debe ser parte de una cultura de la evaluación y convertirse en una práctica cotidiana, capaz de generar cambios positivos en los procesos educativos.

\section{Metodología}

La metodología utilizada para el estudio de caso fue un trabajo de campo de tipo censal. Se seleccionó a los 49 profesores de la carrera de Administración de Empresas del Instituto Superior Tecnológico Edupraxis en Ecuador. A ellos se les aplicó una encuesta de dieciocho preguntas, referidas a la variable "criterios utilizados en la evaluación del desempeño". Estas preguntas fueron de opción múltiple, tipo escala, con cuatro alternativas de respuesta.

La investigación se realizó en tres fases:

I. Descriptiva. Consistió en un estudio de la realidad del fenómeno para lograr una imagen de la situación.

II. Analítica. Comprende dos aspectos: la fundamentación teórica que se logró mediante la revisión bibliográfica y el análi- 
sis e interpretación de resultados, según la estadística porcentual.

III. Diseño. Se formuló una propuesta de criterios para la evaluación del desempeño de profesores de Educación Superior.

\section{Resultados}

En la tabla uno, se presentan los resultados obtenidos dentro de la institución en donde se realizó el caso de estudio para la variable "criterios utilizados en la evaluación del desempeño". Esta se expresa en tres dimensiones con sus respectivos indicadores: académicos, didácticos y logros de los estudiantes.

De las respuestas emitidas en la tabla uno, se desprende que el $56.9 \%$ de los directivos y profesores encuestados consideran que al evaluar el desempeño se consideran los títulos académicos; y según el $43.1 \%$ de los encuestados valoran el hecho que el profesor se actualice permanente. El promedio favorable obtenido (50\%) es indicador de que se asume, parcialmente, la formación profesional como criterio de evaluación del desempeño laboral.
Solo para el $33.5 \%$ se asume como criterio de evaluación la producción intelectual, tales como libros, artículos, ponencias o materiales instruccionales. Asimismo, para el $56.9 \%$ se puntúa la experiencia como criterio de evaluación del desempeño del profesor de educación superior tecnológica. El promedio favorable obtenido para la dimensión (42.8\%) permite inferir que, los criterios académicos sobre la evaluación del desempeño en la institución se toman en cuenta muy poco.

Los resultados presentados en la tabla dos muestran que, según el 59\% de los encuestados en la institución, casi siempre se considera en la evaluación del desempeño los planes de clase o los proyectos elaborados por los profesores. Para el $100 \%$ de estos casi siempre se asume como criterio de evaluación del profesor el cumplimiento de los planes, los métodos y recursos de enseñanza, el plan de evaluación y las técnicas e instrumentos utilizados en la evaluación de los estudiantes.

Tabla 1. Criterios Académicos

\begin{tabular}{|c|c|c|c|}
\hline \multirow{2}{*}{\multicolumn{2}{|c|}{ Indicadores }} & \multicolumn{2}{|c|}{ Alternativas } \\
\hline & & \multirow{2}{*}{$\frac{\text { Favorable }}{56.9 \%}$} & \multirow{2}{*}{$\begin{array}{c}\text { Desfavorable } \\
43.1 \% \\
\end{array}$} \\
\hline Formación & Títulos académicos & & \\
\hline profesional & Actualización docente & $43.1 \%$ & $56.9 \%$ \\
\hline \multirow{2}{*}{$\begin{array}{l}\text { Producción } \\
\text { intelectual }\end{array}$} & $\begin{array}{l}\text { Resultados de investigación: libros, artícu- } \\
\text { los de revistas, ponencias. }\end{array}$ & $33.5 \%$ & $66.5 \%$ \\
\hline & Materiales instruccionales & $33.5 \%$ & $66.5 \%$ \\
\hline $\begin{array}{l}\text { Experiencia } \\
\text { Personal }\end{array}$ & Cinco años de servicio en la asignatura & $56.9 \%$ & $43.1 \%$ \\
\hline \multicolumn{2}{|r|}{ Promedio } & $42.8 \%$ & $57.2 \%$ \\
\hline
\end{tabular}


Tabla 2. Criterios Didácticos

\begin{tabular}{|c|c|c|c|}
\hline \multirow{2}{*}{\multicolumn{2}{|c|}{ Indicadores }} & \multicolumn{2}{|c|}{ Alternativas } \\
\hline & & \multirow{2}{*}{$\begin{array}{c}\text { Favorable } \\
59 \%\end{array}$} & Desfavorable \\
\hline \multirow{8}{*}{$\begin{array}{l}\text { Competencias } \\
\text { metodológicas }\end{array}$} & $\begin{array}{l}\text { En la evaluación del docente se consideran } \\
\text { los planes de clase o proyectos }\end{array}$ & & \multirow{3}{*}{$41 \%$} \\
\hline & El cumplimiento de los planes & $100 \%$ & \\
\hline & Los métodos de enseñanza & $100 \%$ & \\
\hline & Los recursos de aprendizaje & $100 \%$ & \multirow{5}{*}{$0 \%$} \\
\hline & El plan de evaluación & $100 \%$ & \\
\hline & Las técnicas de instrumentos de evaluación & $100 \%$ & \\
\hline & El dominio de la asignatura & $100 \%$ & \\
\hline & El cumplimiento de las normas & $100 \%$ & \\
\hline & Promedio Parcial & $94.4 \%$ & $5.6 \%$ \\
\hline \multirow{2}{*}{$\begin{array}{l}\text { Relaciones con } \\
\text { los alumnos }\end{array}$} & $\begin{array}{l}\text { Se considera en la evaluación del docente } \\
\text { las relaciones con los alumnos }\end{array}$ & $59 \%$ & \multirow{2}{*}{$41 \%$} \\
\hline & $\begin{array}{l}\text { El interés manifestado por el docente hacia } \\
\text { el estudiante }\end{array}$ & $59 \%$ & \\
\hline \multicolumn{2}{|r|}{ Promedio Parcial } & $79.5 \%$ & $20.5 \%$ \\
\hline \multicolumn{2}{|r|}{ Promedio Total } & $86.9 \%$ & $13.7 \%$ \\
\hline
\end{tabular}

Igualmente, el $100 \%$ opina que siempre se considera el dominio de la asignatura y el cumplimiento de los reglamentos de asistencia, puntualidad, así como la sujeción al programa. El porcentaje favorable obtenido (94.4\%) para el indicador competencias, evidencia que las competencias metodológicas del profesor son un criterio importante en su evaluación.

Por su parte, el 59\% considera que casi siempre se considera en la evaluación del profesor las relaciones con los estudiantes, mientras que para el $100 \%$, casi siempre se considera el interés manifestado por el profesor hacia el estudiante. El promedio favorable $(79.5 \%)$ obtenido para esta categoría evidencia que se asume como criterio las relaciones con los estudiantes, pero en menor proporción que las competencias metodológicas del profesor. El promedio favorable $(86.9 \%)$ obtenido, permite inferir que se le asigna un importante peso a los criterios didácticos en la evaluación del desempeño profesoral en la institución.

Los resultados de la tabla tres muestran que, según el $100 \%$ de los encuestados no se considera el porcentaje de estudiantes aprobados, ni las calificaciones promedio en la asignatura que dicta el profesor, ni el logro de los objetivos del programa. Esto evidencia que el promedio favorable en los dos indicadores considerados -y por tanto en la dimensión- es nulo. Se infiere que no se ponderan los logros 
Tabla 3. Logro de los estudiantes como criterio de evaluación del desempeño

\begin{tabular}{|c|c|c|c|}
\hline \multirow{2}{*}{\multicolumn{2}{|c|}{ Indicadores }} & \multicolumn{2}{|c|}{ Alternativas } \\
\hline & & Favorable & Desfavorable \\
\hline \multirow{2}{*}{$\begin{array}{l}\text { Rendimiento } \\
\text { académico }\end{array}$} & El porcentaje de estudiantes aprobado & -- & $100 \%$ \\
\hline & $\begin{array}{l}\text { Las calificaciones promedio en la asignatu- } \\
\text { ra que dicta }\end{array}$ & -- & $100 \%$ \\
\hline $\begin{array}{l}\text { Aprendizaje } \\
\text { logrado }\end{array}$ & El logro de los objetivos de programa & -- & $100 \%$ \\
\hline \multicolumn{2}{|r|}{ Promedio } & -- & $100 \%$ \\
\hline
\end{tabular}

del estudiante como criterio de evaluación del desempeño del profesor.

Al integrar los resultados obtenidos para la variable "criterio de evaluación del desempeño de profesores", se obtiene que existe un porcentaje favorable del $42.8 \%$ para los criterios académicos y un $86.9 \%$ para los criterios didácticos; pero para los logros de los estudiantes existe un porcentaje nulo.

El promedio favorable (43.2\%) obtenido para la variable, permite inferir las debilidades en cuanto a los criterios utilizados para la evaluación del desempeño ya que, si bien se les da una alta importancia a los criterios didácticos, no ocurre así con los académicos y menos con los logros de los estudiantes. Con base a los resultados, se proponen algunos elementos para la evaluación del desempeño profesoral en educación tecnológica.

\section{Discusión}

Los resultados del estudio muestran que no existe un verdadero sistema de evaluación del desempeño docente; sin embargo, esta práctica se hace, principalmente, a través de cuestionarios aplicados a los estudiantes, los cuales son elaborados por autoridades administrativas. No hay procedimientos claramente establecidos acerca de cómo interpretar la información, ni qué utilidad tendrán los datos. Tales resultados coinciden con los de Parada (2016), en cuanto que la evaluación en las instituciones de educación superior tecnológica es instrumentalista, cuantitativa y sumativa; apoyada en un solo cuestionario, diligenciado por los estudiantes. Este es un requisito que se limita solo al papel, porque es un proceso de rendición de cuenta y no formativo.

Al respecto, Arias (2015) plantea que la evaluación del desempeño docente -cualquiera que sea el método- es compleja y difícil; dentro de la cual existen una serie de métodos, cada uno con sus pros y sus contras. No obstante, señala que no se puede omitir la observación de los docentes en su acción, por lo que se deben incorporar fuentes múltiples. Montoya (2014) plantea que se debe tratar de abordar de manera integral las diferentes 
dimensiones, voces y contextos relacionados con la actividad de los profesores universitarios, a fin de obtener un panorama completo de la docencia. En este sentido, recomiendan incluir métodos cualitativos.

La evaluación del desempeño laboral es un aspecto fundamental del proceso de gestión del talento humano, por cuanto la principal ventaja competitiva de las instituciones superiores se deriva de los profesores que laboran en estas. Actúa como función de control y su finalidad última es la mejora de la organización, a través de la mejora continua de las personas; su utilidad radica en ser un instrumento para planificar dicha mejora. Al respecto, Tejedor (2011) plantea que la función de la evaluación del desempeño docente es doble: a) como instrumento técnico de control, de responsabilidad y de imputabilidad; y b) como instrumento de formación de los profesores con respecto a su misión con la sociedad.

La evaluación del desempeño es una apreciación sistemática del trabajo de cada persona, en función de las actividades que cumple, de las metas y resultados que debe alcanzar y de su potencial de desarrollo. De ahí que las evaluaciones son necesarias para contar con una buena gestión y desarrollar el talento humano. Sin embargo, este proceso debe ser planeado para garantizar que la evaluación pueda hacerse de manera realista dentro de las características de la institución. En consecuencia, la elaboración del plan debe ser un proceso interactivo, en el cual se especifiquen los papeles y responsabilidades de la persona evaluada y del evaluador.

Pacheco y otros (2018) plantean que para realizar una evaluación del desempeño es indispensable delimitar el perfil del buen docente en el contexto institucional; es decir, deben definirse los conocimientos, habilidades y actitudes (competencias) que idealmente tendría que dominar un profesor; las condiciones de corte disciplinar, las necesidades de evaluación y uso que se darán a los resultados, configurando el perfil particular de una determinada institución.

En tal sentido, los criterios que se proponen están orientados a optimizar la evaluación del desempeño del profesor de Institutos Superiores de Tecnología, los cuales resolverían el problema planteado en el área de la gestión del talento humano como, por ejemplo, optimizar el desempeño; y por la otra, minimizar las debilidades encontradas en la evaluación del desempeño que se realiza en la actualidad. Esto permitirá entre otros aspectos, tener el profesor adecuado para los estudios de tecnología que requieren la estructura organizativa; pudiendo -al mismo tiempo- planificar de manera oportuna, y coherente el desarrollo del personal, según sus necesidades. Todo esto le permitirá garantizarles su trayectoria académica en la institución, obligándole a re conceptualizar la evaluación del desempeño del profesor universitario.

De ahí que la revisión de las bases científicas más recientes muestra la necesidad de un proceso de evaluación, que complemente la 
autoevaluación con los aportes de otros actores: directivo, colegas, comunidad; y de esta manera evitar sesgos y ofrecer oportunidad al docente de revisar aspectos que desde su posición no son observables.

Hacer funcionar la evaluación del desempeño requiere que se cumpla con cuatro fases:

1. La fase informativa requiere divulgar informaciones sobre sus objetivos y funcionamiento. Así como la promoción de sus beneficios y ventajas.

2. La fase motivacional tiene como objetivo reflexionar para sensibilizar a los involucrados en el proceso. Para lograr esto se cumplirán dos procedimientos: (a) establecimiento de un plan de incentivos, que motive al profesor hacia la obtención de sus objetivos y el cumplimiento de sus actividades; y (b) definir las acciones para motivar y desarrollar a los profesores.

En tal sentido, los incentivos que se proponen son:

- Bonificación especial por méritos. Concedido a aquellos profesores que resulten sobresalientes en su evaluación de desempeño.

- Compensación por conocimientos especializados. Este incentivo no se hará con base en lo que hace el profesor con lo que sabe, sino en lo que puede hacer.

- Otras formas de reconocimiento, tales como: condecoraciones, felicitaciones, botones por tiempo de servicio, pagos de horas extras; que sirven para alentar esfuerzos adicionales dirigidos a un propósito específico. Entre las acciones para motivar y lograr el desarrollo profesional de profesores, se pueden mencionar: obtención de mejores de niveles de desempeño; oportunidades de ascensos, cuando los profesores mejoran sus calificaciones $y$ complementan los objetivos de la organización. Tanto como la experiencia en nuevos puestos como la obtención de nuevos conocimientos y habilidades constituyen vehículos para el crecimiento profesional.

3. La fase de aplicación implica -en primer lugar- establecer el compromiso, los roles y la responsabilidad, tanto de evaluadores como de evaluados y socios en la actividad.

4. La fase de seguimiento se producirá durante todo el proceso, y permitirá ir incorporando los correctivos hasta que el proceso esté listo para su aplicación permanente.

Entre estos requerimientos para la evaluación, se debe contar con el total conocimiento y plena comprensión del proceso por parte de toda la comunidad que integra la institución; la conformación del equipo evaluador para la recolección de información y seguimiento al plan de desarrollo profesional. También, con el conocimiento de los instrumentos y la metodología por parte del equipo evaluador y los evaluados; organización del proceso para el lapso académico, la definición y concertación de los criterios, y los instrumentos necesarios para la recolección de la información. Así como del seguimiento a los compromisos, el registro de las observaciones, diálogos, reu- 
niones y demás actividades en torno a la evaluación de desempeño y el plan de desarrollo.

El desarrollo de la evaluación del desempeño es un proceso sistemático que se lleva a cabo durante todo el año académico e implica:

- La recolección de información, la identificación de evidencias válidas y confiables.

- La valoración de las evidencias y el plan de desarrollo.

La información puede provenir de fuentes personales, integrantes de la comunidad educativa o cuerpos colegiados de la institución que puedan dar testimonio sobre el desempeño del evaluado. Documentos, registros y testimonios escritos -personales e institucionales- como: innovaciones educativas, resultados significativos en los aprendizajes de los estudiantes; desarrollo de proyectos, actas, programaciones e informes. Además, son fuentes personales: el coordinador, el par académico, los estudiantes, el directivo y el propio evaluado y su autoevaluación.

Las observaciones pueden ser una gran fuente de información, siempre y cuando se preparen para determinar qué se va a observar, cuándo, durante qué tiempo y en el marco de qué aspectos del desempeño. Esta herramienta es la oportunidad para precisar algunas de las informaciones ya recogidas; conversar con el evaluado, retroalimentar, revisar y priorizar algunos requerimientos de desarrollo profesional.
La información solicitada a las fuentes personales o recogida en las observaciones, debe registrarse en formatos diseñados para tal fin: registros de información, cuestionarios, diarios de campo y otros similares. Para cada profesor debe organizarse un portafolio o carpeta personal, en la cual se van guardando todas las evidencias y registros de las informaciones obtenidas, de manera secuencial, con fechas y datos precisos en cada uno de estos.

De acuerdo a Pacheco y otros (2018), la evaluación del desempeño docente -a través del portafolio- exige un proceso de integración de evidencias y reflexiones organizadas, a partir de un conjunto de criterios establecidos por los líderes del proceso evaluativo, que permitirá juzgar su desempeño. Este modelo tiene capacidad para combinar aspectos cuantitativos y cualitativos; así como para incorporar resultados de otras evaluaciones, por lo que se presenta como un modelo más integrador. Esta información se convierte en evidencias que hacen referencia a una serie de productos o demostraciones requeridas para probar el desempeño en un aspecto específico del profesor que se va a evaluar. Las evidencias son claves para soportar la valoración que el evaluador le da, posteriormente, a los diferentes aspectos evaluados y que consigna en el instrumento de valoración.

La información que se ha recogido durante el año, se organiza de acuerdo con los aspectos que se evalúan y con los compromisos establecidos en el plan de desarrollo profesional. 
La información proveniente de diferentes fuentes y en diferentes momentos o situaciones se analiza, se cruza entre sí y -si es necesario- se triangula para encontrar concordancias o discrepancias.

Al final del año, después de recoger la información y tener evidencias y elementos para emitir un juicio valorativo sobre el desempeño, en una entrevista programada se analizan en conjunto los aspectos evaluados, y el evaluador emite su juicio valorativo; consigna en el instrumento de evaluación el valor asignado a cada aspecto y elabora con el evaluado el plan de desarrollo profesional.

Esta sesión de valoración es uno de los elementos más fructíferos del proceso de evaluación del desempeño. Debe ser la oportunidad ideal para explorar con el evaluado -en un ambiente amable, tranquilo y respetuoso- su desempeño, el cumplimiento de responsabilidades y los compromisos adquiridos para un buen trabajo; así como los aspectos de posible mejoramiento, aprendizaje y cambio. También debe ser la oportunidad para identificar y comprender los sentimientos y actitudes del evaluado frente a su trabajo.

Para que el evaluado logre una actitud abierta al cambio y de aceptación a las sugerencias y observaciones hechas por el evaluador, es necesario que el evaluado:

- No se sienta amenazado durante su desarrollo.

- Tenga la oportunidad de presentar ideas y sentimientos.
- Cuente con un evaluador que se centre en los aspectos positivos del trabajo; que enfatice en los puntos fuertes sobre los cuales puede apoyarse.

- Encuentre una atmósfera donde la conversación se caracteriza por el respeto mutuo, la sinceridad, la actitud de escucha y el flujo de información en los dos sentidos.

Sin embargo, como no se desea solamente que los evaluados se sientan satisfechos con la entrevista, sino que se cumpla con el objetivo de estimular el desarrollo del evaluado discutiendo los problemas, las necesidades, las innovaciones, las insatisfacciones y las inconformidades que se hayan encontrado en el desempeño del trabajo, el evaluador debe ser claro, directo y específico para señalar los puntos débiles. Se debe evitar referirse a situaciones vagas y a la generalización de un comportamiento problemático.

Esta claridad facilita la construcción del plan de desarrollo profesional, al cual se llevan los aspectos más importantes que sean posibles de trabajar dentro de un periodo razonable. Para lograr que el proceso de evaluación de desempeño cumpla los fines y objetivos previstos, se sugiere diseñar un instrumento que conste de dos partes: valoración anual del desempeño y plan de desarrollo profesional.

La parte de valoración anual del desempeño debe ser diligenciado por el superior inmediato al final del año lectivo, en un diálogo programado con el evaluado y con toda la información necesaria, recogida durante ese año. Se 
sugiere la evaluación del profesor, tomando en consideración los siguientes aspectos: cumplimiento de normas y políticas institucionales, conocimiento y valoración de los estudiantes; fundamentación pedagógica, planeación del trabajo, estrategias didácticas, estrategias para la participación, evaluación y mejoramiento; innovación, compromiso institucional, relaciones interpersonales, mediación de conflictos, trabajo en equipo y liderazgo

En la misma sesión, el evaluado y el evaluador identifican los aspectos que serán objeto de mejoramiento específico durante el periodo académico siguiente. Para estos aspectos (se recomienda un número máximo de dos), se establecen compromisos concretos y medibles, expresados en metas, acciones realistas y tiempos precisos, que serán objeto de seguimiento. La definición clara de estos aspectos permitirá en buena medida el éxito del seguimiento al plan de desarrollo.

Así, los aspectos de mejoramiento deben señalarse como nuevos retos para avanzar en la formación personal y profesional; las fortalezas se deben reconocer y valorar como aspectos de enriquecimiento profesional e institucional. Todos los profesores presentan aspectos que pueden ser objeto de avance y perfeccionamiento, por eso no solo se prestará especial atención a aquellos aspectos que muestren un desempeño de nivel inferior.

En el plan de desarrollo profesional deben establecerse momentos de seguimiento para analizar los cambios, registrar los avances alcanzados y acordar -si se considera nece- sario- nuevos momentos de entrevista. Estos momentos de seguimiento, dependen de las exigencias del plan. Todos los evaluados deben tener al menos dos entrevistas con el evaluador durante el año para conocer su desempeño, sus avances y nuevas propuestas.

Es deseable que exista un compromiso conjunto, tanto de aquellos que se han desempeñado en un nivel superior como aquellos que tienen una evaluación de nivel medio o inferior. De esta manera es posible construir una organización que aprende, en aras de perfeccionar y/o mejorar la calidad del proceso de educación desde una perspectiva más integradora.

Al concluir, es importante señalar que el tema de evaluación del desempeño del profesor universitario tiene mucho que aportar aún al campo de la investigación. En primer lugar, porque la labor docente es compleja, pero esencial en el éxito del proceso educativo. Esto implica que es un proceso no totalmente objetivo, ni fundamentado como se quisiera. Por otra parte, implica considerar un conjunto de elementos que no se conocen suficientemente todavía. Específicamente, la evaluación del profesor en las Instituciones de Educación Superior Tecnológica todavía puede considerarse incipiente. Lo que tiene sentido, en cuanto brinda la oportunidad de abrir nuevos horizontes, un nuevo estilo de reflexión sobre la práctica educativa y perfil del profesor universitario. 


\section{Referencias}

Álvarez, C. (2003). Un sistema de autoevaluación y mejora para el profesorado de Educación. Recursos e Instrumentos Psicopedagógicos. Bilbao: Ediciones Mensajero.

Arias, G. (2015). Evaluación del desempeño profesional de formadores de docentes en El Salvador (Tesis doctoral). Mejilla, España: Universidad de Granada.

Asamblea, N. (2010). Ley Orgánica de Educación Superior. Quito

Barceló, M. (1998). La sociedad del conocimiento. Barcelona, España: Beta.

Barreto, C. (2012). Evaluación de la calidad del desempeño y directivo en el Instituto Superior Agropecuario José Benigno Iglesias de la parroquia Biblian, de la Provincia de Cañar, durante el año académico 2011-2012. Loja, Ecuador. Centro Universitarios Cuenca.

Barreto, I. (2015). Desempeño docente y desarrollo de las capacidades en gestión empresarial y marketing en estudiantes de la Escuela Superior Tecnológica-Sencico (Tesis doctoral). Lima, Perú: Universidad San Martín de Porres.

Bell, D. (1991). El advenimiento de la sociedad post-industrial. Madrid: Alianza

Chaverry, R. (2009). Propuesta alternativa de evaluación del desempeño docente universitario. Recurado de http:// www: portalesmedicos.com /.../propuesta-alternativa-de-eval

Chiavenato, I. (2004). Gestión del Talento Humano. Colombia: Mac Graw Hill

Consejo de Educación Superior República de Ecuador (2012). Reglamento de Carrera y Escalafón del Docente e Investigador del Sistema de Educación Superior. Quito

Coppola, N. (2012). Las políticas de evaluación de la función docente universitaria en el marco de la evaluación institucional. Un estudio comparado entre Argentina, España y México (Tesis doctoral). Madrid: Universidad Complutense.

Davis, R. (1992). Evaluación del Desempeño Docente. Recuperado de imágenes buena-docencia multiply.

Drucker, P. (1993). La sociedad poscapitalista. Buenos Aires: Sudamericana

Estrada, L. (2012). El desempeño docente. Venezuela: UC

Gallardo, L. y Zampella, A. (2001). Postgrado de Recursos Humanos: acortando la brecha entre el aula y la realidad. Revista Recursos Humanos, 3 (10). Venezuela: Asociación de Derechos Humanos

García, J y otros (2005). Fundamentos de Dirección y Gestión de Recursos Humanos. Madrid: Thomson. 
Hargreaves, A. (2007). Enseñar en la sociedad del conocimiento. La educación en la era de la inventiva. Barcelona: Octaedro

Hidalgo, C. (2007). Retos y Desafíos de la Evaluación Docente. Congreso Internacional de Evaluación de la función Docente. Recuperado de http:// congreso educación e/programa.html

Lawler III, E. (2000). Cómo recompensar la excelencia. Colombia: Editorial Norma.

Lima, B. (2017). Evaluación del desempeño docente y su relación con el rendimiento académico de los estudiantes de la carrera de Ingeniería Metalúrgica y Materiales (UMSA). (Tesis de Maestría). La Paz, Bolivia: CEPIES-UMSA

López, M. (2014). Evaluación del desempeño docente de la Carrera de ingeniería Ambiental de la Facultad de Ciencias Naturales, Universidad de Guayaquil, frente al Reglamento de Escalafón y Carrera Docente. Propuesta de un Sistema de Evaluación Docente Alternativo (Tesis de Maestría). Quito: Universidad de Guayaquil.

Marcano, L. (2008). Modelo de Evaluación del Desempeño Profesional Docente. Cuba: Editorial Universitaria

Martínez-Izaguirre, M; Yániz-Álvarez, C y Villardón-Gallego, L. (2018). Autoevaluación y reflexión docente para la mejora de la competencia profesional del profesorado en la sociedad del conocimiento. RED Revista de Educación a Distancia, 56. Disponible: http://www. um.es/ead/red/...pdf

Montoya, J y otros (2014). Evaluación de la docencia universitaria en México, Chile y Colombia: análisis de experiencias. Revista Iberoamericana de Evaluación Educativa 7(2e), 15-42.

Mora, D. (2004). Nuevo Rol del Docente del Siglo XXI. Recuperado de http:// gestropolis.com/.../ consulta 2010

Pacheco, C y otros (2018). La evaluación del desempeño docente en educación superior. Revista Digital Universitaria 19 (6). Universidad Autónoma de México. DOI: http://doi. org/10.2220v.codeic

Parada, B. (2016). Sistema de evaluación docente, Instituciones de Educación Superior Tecnológica: Lineamientos de calidad. Praxis\&Saber Revista de Investigación y Pedagogía 7 (13), 177-198. UPTC

Tedesco, J. (1999). La educación y la construcción de la democracia en la sociedad del conocimiento. Buenos Aires: IIPE-UNESCO

Touraine, A. (1973). La sociedad post-industrial. Barcelona: Ariel 